\title{
EFEITO AGUDO DOS EXERCÍCIOS INTERMITENTES SOBRE A GLICEMIA DE ADOLESCENTES COM DIABETES TIPO 1
}

\section{ACUTE EFFECT OF INTERMITTENT EXERCISE ON BLOOD GLUCOSE OF ADOLESCENTS WITH DIABETES TYPE 1}

\author{
EFECTO AGUDO DE EJERCICIOS INTERMITENTES SOBRE LA GLUCEMIA EN ADOLESCENTES CON \\ DIABETES TIPO 1
}

Valderi Abreu de Lima' (Profissional de Educação Física) Luis Paulo Gomes Mascarenhas ${ }^{2}$ (Profissional de Educação Física) Juliana Pereira Decimo' (Profissional de Educação Física) William Cordeiro de Souza ${ }^{3}$ (Profissional de Educação Física) Suzana Nesi França ${ }^{1}$ (Médica Endocrinologista) Neiva Leite ${ }^{1}$ (Profissional de Educação Física, Médica Pediatra)

1. Universidade Federal do Paraná Curitiba, PR, Brasil.

2. Universidade Estadual do Centro Oeste. Irati, PR, Brasil.

3. Universidade do Contestado. Canoinhas, SC, Brasil.

\section{Correspondência:}

R. Arthur Virmond de Lacerda, 36, Jardim Montreal, Lapa, PR, Brasil. 83750-000.

valderiabreulima@hotmail.com

\section{RESUMO}

Introdução: O exercício físico é um fator importante do tratamento do diabetes mellitus. Objetivo:Verificar o efeito agudo dos exercícios intermitentes sobre glicemia e oxidação de substratos energéticos em adolescentes com diabetes tipo 1. Métodos: Foram avaliados 10 adolescentes diabéticos com idade entre 10 e 15 anos. Foram avaliadas as medidas antropométricas, hemoglobina glicosilada $(\mathrm{HbA1c}), \mathrm{VO}_{2 \text { máx }}$ e o teste de exercício intermitente. Nesse teste, os indivíduos permaneceram pedalando por 30 minutos em cicloergômetro com carga de $60 \%$ do $\mathrm{VO}_{2 \text { máx }}$ intercalados com tiros de intensidades máximas de 10 segundos a cada 5 minutos. Os substratos energéticos foram avaliados durante o teste e a glicemia capilar foi medida antes, imediatamente após o exercício e 30 minutos depois. Foi observada redução média da glicemia de $39,2 \pm 41,92 \mathrm{mg} / \mathrm{dl}$, isto é, média de $21,61 \%$ da glicemia inicial. Ocorreu diferença significativa $(0,0001)$ entre os substratos energéticos oxidados, com predominância da utilização de CHO. Há uma forte correlação direta entre a HbA1c e o escore $z$ do IMC ( $r=0,821, p=0,004)$ e entre HbA1 c e glicose observadas pós-exercício $(r=0,702, p=0,024)$ e também há forte correlação entre os níveis de glicose pré-exercício e pós-exercício $(r=0,851, p=0,002)$ e entre pós-exercício e 30 minutos depois do exercício $(r=0,874, p=0,001)$. $O$ teste de regressão linear mostrou que o escore $z$ do IMC explica 67\% dos valores de HbA1c $\left(r^{2}=0,675\right)$. Resultados: De acordo com os resultados observados, 30 minutos de exercícios aeróbicos intermitentes intercalados com tiros curtos de 10 segundos promovem redução média de $21 \%$ da glicemia, com tendência de aumento na fase de recuperação. O substrato energético predominante na atividade são os carboidratos (CHO). Conclusão: Quanto melhor for o escore z do IMC do adolescente, espera-se melhor controle glicêmico em adolescentes com diabetes tipo 1.

Descritores: metabolismo dos carboidratos; diabetes mellitus; oxidação.

\section{ABSTRACT}

Introduction: Physical exercise is an important factor in the treatment of diabetes mellitus. Objective: To verify the acute effect of intermittent exercises on blood glucose and oxidation of energetic substrates in adolescents with type 1 diabetes. Methods: We evaluated 10 diabetic adolescents aged 10 to 15 years. Anthropometric measurements, glycosylated hemoglobin $(\mathrm{HbATC}), \mathrm{VO}_{2 \max }$ and the intermittent exercises test were evaluated. In this test, subjects continued pedaling for 30 minutes on a cycle ergometer with a load of $60 \%$ of $\mathrm{VO}_{2 \text { max }}$ interspersed with maximum intensity sprints of 10 seconds every 5 minutes. The energetic substrates were evaluated during the test and the capillary blood glucose was measured before, immediately after exercise, and 30 minutes later. We observed a mean reduction in blood glucose of $39.2 \pm 41.92 \mathrm{mg} / \mathrm{dl}$, that is, an average of $21.61 \%$ of initial blood glucose. There was a significant difference $(0.0001)$ between the oxidized energetic substrates, predominating the use of $\mathrm{CHO}$. There is a strong direct correlation between $\mathrm{HbAlc}$ and the BMI z score $(r=0.821, p=0.004)$ and between HbA1c and glucose observed post-exercise $(r=0.702, p=0.024)$, and there is a strong correlation between pre-exercise and post-exercise glucose $(r=0.851, p=0.002)$ and between post-exercise and 30 minutes after exercise $(r=0.874, p=0.001)$. The linear regression test showed that $B M I z$ score accounts for $67 \%$ of the HbA1c values $\left(r^{2}=0.675\right)$. Results: According to the results observed, 30 minutes of intermittent aerobic exercises interspersed with short sprints of 10 seconds promoted a mean reduction of $21 \%$ in blood glucose with tendency to increase in the recovery phase. The predominant energetic substrates in the activity are carbohydrates (CHO). Conclusion: The better the adolescent BMI zscore, the better glycemic control in adolescents with type 1 diabetes is expected.

Keywords: carbohydrate metabolism; diabetes mellitus; oxidation.

\section{RESUMEN}

Introducción: El ejercicio es un factor importante en el control de la diabetes. Objetivo: Investigar el efecto agudo de ejercicio intermitente en la glucemia y la oxidación de sustratos energéticos en adolescentes con diabetes tipo 1. Métodos: Se estudiaron 10 adolescentes diabéticos de 10 a 15 años. Se evaluaron las medidas antropométricas, la hemoglobina glucosilada (HbA1c), el VO ${ }_{2 \text { máx }}$ y la prueba de ejercicio intermitente. En esta prueba, los individuos permanecieron pedaleando durante 30 minutos en un cicloergómetro con carga del $60 \%$ del $\mathrm{VO}_{2 \operatorname{máx}}$ intercalados con piques de máxima intensidad de 10 segundos cada 5 minutos. Se evaluaron sustratos energéticos durante la prueba y la glucemia capilar fue medida antes, inmediatamente después del ejercicio y 30 minutos más tarde. Se observó 
una reducción promedio de la glucemia de 39,2 $\pm 41,92 \mathrm{mg} / \mathrm{dl}$, es decir, promedio de 21,61\% sobre la glucemia inicial. Hubo una diferencia significativa $(0,0001)$ entre los sustratos energéticos oxidados, predominando el uso de CHO. Hay una fuerte correlación directa entre la HbA1cy el IMC puntuación z ( $r=0,821, p=0,004)$ y entre HbA1cy glucosa observadas después del ejercicio $(r=0,702, p=0,024)$ y también hay una fuerte correlación entre los niveles de glucosa pre-ejercicio y post-ejercicio $(r=0,851, p=0,002)$ y post-ejercicio y 30 minutos después del ejercicio $(r=0,874, p=0,001)$. La prueba de regresión lineal mostró que el IMC puntuación zexplica el $67 \%$ de los valores de HbAIC $\left(R^{2}=0,675\right)$. Resultados: De acuerdo con los resultados observados, 30 minutos de ejercicio aeróbico intermitente intercalado con piques cortos de 10 segundos promueven una reducción promedio del $21 \%$ de glucemia, con una tendencia al aumento en la fase de recuperación. El sustrato energético predominante en la actividad son los carbohidratos (CHO). Conclusión: Cuanto mejor sea la puntuación $z$ del IMC de los adolescentes, mejor control de la glucemia se puede esperar en adolescentes con diabetes tipo 1.

Descriptores: metabolismo de los hidratos de carbono; diabetes mellitus; oxidación.

\section{INTRODUÇÃO}

O exercício físico é um fator importante do tratamento do diabetes mellitus, e contribui para melhorar a qualidade de vida, atuando preventivamente nos riscos de complicações associadas como nefropatias, neuropatias, retinopatias e doenças cardiovasculares ${ }^{1}$.

Dentre os benefícios em curto prazo dos exercícios podemos citar o aumento do consumo de glicose como substrato energético pelos músculos em atividade, esse efeito hipoglicemiante pode se prolongar por horas após a atividade ${ }^{1}$.

Todavia as taxas de utilização de glicose variam dependendo do tipo, intensidade e duração do exercício, o efeito pode ser hipoglicemiante ou até mesmo hiperglicemiante, devido a ação e secreção de hormônios contrarreguladores ${ }^{2,3}$.

Os diversos tipos de exercícios influenciam na regulação do metabolismo dos carboidratos e gorduras que sofrem complexas modificações ${ }^{2,4}$.

Exercícios aeróbicos contínuos de baixa intensidade podem favorecer uma redução rápida da glicemia em diabéticos tipo 1, podendo levar a uma hipoglicemia induzida pelo exercício ${ }^{3}$. Por outro lado, a resposta glicêmica aos exercícios intermitentes de alta intensidade é pouco compreendida, este padrão de atividade envolve sprints curtos e intensos com períodos de baixa intensidade ou descanso, característica da maioria dos esportes de equipe e jogos espontâneos de crianças 5,6. Alguns trabalhos demonstram que exercícios intermitentes de alta intensidade parecem estar associados a uma redução dos riscos de hipoglicemias pós-exercício 7,8. Porém a maioria destes estudos foi realizada com a população adulta.

Devido à carência de estudos que avaliem as respostas dos exercícios intermitentes em adolescentes, o objetivo deste estudo foi de investigar o efeito agudo dos exercícios intermitentes na resposta glicêmica, e a participação de carboidratos $(\mathrm{CHO})$ e gorduras como fontes energéticas de adolescentes diabéticos tipo 1.

\section{MATERIAL E MÉTODO}

A presente pesquisa descritiva de corte transversal avaliou 10 adolescentes com diabetes mellitus tipo 1, atendidos no ambulatório de Diabetes da Unidade de Endocrinologia Pediátrica do Hospital de Clínicas de Curitiba (HCC), Curitiba, PR, Brasil.

A amostra foi selecionada por conveniência, avaliando-se os pacientes que aceitaram participar da pesquisa e com autorização dos pais ou responsáveis, mediante a apresentação do Termo de Consentimento Livre e Esclarecido, de acordo com a Resolução 196/96 do Conselho Nacional de Saúde. Aprovado pelo Comitê de Ética em Pesquisa da Universidade Federal do Paraná, Curitiba, PR, Brasil, sob o número 44193214-7.0000.0096.

Foram incluídos adolescentes com diagnóstico de diabetes há no mínimo dois anos, com idades entre 10 e 15 anos e sem outras comorbidades associadas. Foram excluídos do estudo pacientes fora da faixa etária, que apresentassem alterações ortopédicas ou outras comorbidades que impossibilitassem a realização de atividade física.

Para obtenção das medidas antropométricas foram utilizadas técnicas conforme descrito por Lohman9. A estatura foi mensurada com estadiômetro vertical portátil (WCS ${ }^{\circledR}$, Brasil), escalonado em 0,1 e avaliado em centímetros (cm), ao final de inspiração máxima. A massa corporal foi aferida em balança digital portátil (Filizola ${ }^{\circledR}$, Brasil), em quilogramas (kg). Em seguida foi calculado o índice de massa corporal $(\mathrm{kg} / \mathrm{m} 2)$ pela divisão da massa corporal pela estatura ao quadrado, e convertido para IMC-escore Z.

A avaliação do controle glicêmico foi realizada por meio dos níveis de hemoglobina glicada (HbA1c) avaliado por teste imunoturbidimétrico TurbiClin (São Paulo - Brasil), um jejum prévio de 12 horas o avaliado realizou a punção venosa sanguínea, o sangue então foi levado para análise Cromatográfica.

Exames laboratoriais foram realizados após a realização dos testes, foram coletados $8 \mathrm{ml}$ de sangue por punção venosa para a dosagem dos níveis de cortisol e insulina.

Os avaliados visitaram duas vezes o laboratório, a primeira para realização do teste da aptidão cardiorrespiratória $\left(\mathrm{VO}_{2 \text { máx }}\right)$ por meio do analisador de gases portátil K4b2 ${ }^{\circledR}$ (Itália) utilizando o protocolo adaptado de Balke em cicloergômetro ${ }^{10}$ que consiste em iniciar o teste com uma carga de 25 Watts e velocidade de 50 RPM, a cada três minutos aumenta-se mais 25 Watts, e assim sucessivamente até ser atingida a frequência cardíaca máxima do indivíduo proposta por Tanaka et al. ${ }^{11}$, ou assim que o mesmo não consiga manter a velocidade e carga.

Após 48 h os avaliados retornaram ao laboratório para a realização do teste de exercício intermitente onde o indivíduo permaneceu pedalando por 30 minutos em cicloergômetro com a carga de $60 \%$ do $\mathrm{VO}_{2 \text { máx }}$ intercalando com cinco tiros de intensidades máximas com duração de 10 segundos a cada 5 minutos Os substratos energéticos oxidados (carboidratos ou gorduras) foram avaliados durante todo o teste por meio do analisador de gases portátil K4b2 ${ }^{\circledR}$ (Itália) e a glicemia capilar mensurada antes, após o exercício e 30 minutos após o término do teste com um glicosímetro Accu-Check, Roche ${ }^{\circledR}$ - Alemanha). A insulinoterapia dos avaliados não foi alterado com doses médias de 26,5 \pm 7,36 de insulina de ação longa Glargina (Brasil) e doses de 7,5 \pm 3,41 de insulina de ação ultra rápida Aspart e Lispro (Brasil).

Todos os testes ocorreram no período da tarde, 1 h após a realização do almoço e aplicação da insulina. O almoço foi padronizado individualmente por nutricionista, com uma porção entre 30 e 35\% da necessidade calórica diária e destes 50 a 55\% foi composto de carboidratos de acordo com a RDA ${ }^{12}$.

A análise estatística foi realizada por meio do programa SPSS for Windows, versão 22 (USA). Para os dados de caracterização da amostra foi utilizada estatística descritiva com média e desvio padrão. Para as variáveis de variação glicêmica e substratos oxidados foi realizado o teste $t$ 
de Student. Utilizou-se a correlação de Pearson para as variáveis HbA1c, $V \mathrm{O}_{2 \mathrm{xa}}$ I IMC escore $\mathrm{z}$, glicose pós-exercícios, percentual de carboidrato oxidado e percentual de gordura oxidada. Para todas as análises utilizou-se nível de significância $p<0,05$.

\section{RESULTADOS}

Os resultados da caracterização da amostra estão descritos na Tabela 1.

Após a realização dos exercícios se observou uma redução média da glicemia de 39,2 —41,92 mg/dl, uma diminuição média de 21,61\% da glicemia inicial. Os valores médios pré, imediatamente após e 30 minutos após o término do exercício podem ser observados na Tabela 2.

Foi encontrada diferença significativa $(0,0001)$ entre os substratos energéticos oxidados durante o exercício (CHOox e GORDox), com uma tendência predominante de oxidação de $\mathrm{CHO}$, desde os primeiros 5 minutos até o término do exercício, como demonstrado na Tabela 3 e Figura 1.

A análise por meio da correlação de Pearson demonstrada na Tabela 4 demonstra uma correlação positiva forte entre a HbA1c e o IMC escore $z(r=0,821, p=0,004)$ e entre HbA1c e glicose observada pós exercício $(r=0,702, p=0,024)$. Podemos ver também uma correlação forte entre os níveis de glicose pré-exercício e pós-exercício $(r=0,851, p=0,002)$ e entre os valores pós-exercício e 30 minutos após o término do exercício $(r=0,874, p=0,001)$. O teste de regressão linear mostrou que o IMC escore z explica $67 \%$ dos valores de HbA1c $\left(r^{2}=0,675\right)$.

\section{DISCUSSÃO}

Os resultados demonstraram uma diminuição media de $21 \%$ da glicemia inicial ( $p=0,014)$, valores médios de 39,2 $\pm 41,92 \mathrm{mg} / \mathrm{dl}$, então se o adolescente iniciar os exercícios intermitentes com a glicemia entre os limites normais ${ }^{1}$, sugere-se, de acordo com os dados observados, que os tiros curtos de alta intensidade realizados por 10 segundos a cada 5 minutos, podem diminuir o risco agudo de hipoglicemia induzida pelo exercício.

Tabela 1. Caracterização da amostra.

\begin{tabular}{c|c}
\hline & Média \pm DP \\
\hline N amostral & 10 \\
\hline Idade (anos) & $13,64 \pm 2,01$ \\
\hline Massa $(\mathrm{kg})$ & $51,62 \pm 14,47$ \\
\hline Estatura $(\mathrm{cm})$ & $156,1 \pm 14,64$ \\
\hline HbAlc $(\%)$ & $9,39 \pm 1,25$ \\
\hline IMC score Z & $0,48 \pm 0,77$ \\
\hline $\mathrm{VO}_{2 \text { máx }}(\mathrm{ml} / \mathrm{min} / \mathrm{kg})$ & $39,05 \pm 8,22$ \\
\hline
\end{tabular}

Tabela 2. Comparação das glicemias pré, pós e 30 min após o término do exercício.

\begin{tabular}{c|c|c|c}
\hline & Média \pm DP & T & $P$ \\
\hline Glicemia pré $(\mathrm{mg} / \mathrm{dl})$ & $\begin{array}{l}181,40 \pm 76,30 \\
142,20 \pm 57,20\end{array}$ & 3,036 & \multirow{2}{*}{$0,014^{*}$} \\
Glicemia pós $(\mathrm{mg} / \mathrm{dl})$ & $\begin{array}{l}181,40 \pm 76,30 \\
159,20 \pm 60,83\end{array}$ & 1,10 & 0,299 \\
\hline Glicemia pré $(\mathrm{mg} / \mathrm{dl})$ & $142,20 \pm 57,20$ & $-1,79$ & 0,106 \\
Glicemia após 30min $(\mathrm{mg} / \mathrm{dl})$ & $159,20 \pm 60,83$ & & \\
\hline Glicemia pós $(\mathrm{mg} / \mathrm{dl})$ & &
\end{tabular}

Tabela 3. Substratos oxidados.

\begin{tabular}{|c|c|c|c|}
\hline & Média \pm DP & $\mathrm{T}$ & $P$ \\
\hline $\begin{array}{l}\text { CHOox (\%) } \\
\text { GORDox }(\%)\end{array}$ & $\begin{array}{l}76,37 \pm 14,42 \\
24,05 \pm 14,54 \\
\end{array}$ & 8,076 & $0,0001^{*}$ \\
\hline $\begin{array}{l}\text { CHOox } 5 \min (\%) \\
\text { CHOox30min (\%) }\end{array}$ & $\begin{array}{l}83,75 \pm 10,59 \\
87,48 \pm 11,32 \\
\end{array}$ & $-0,859$ & 0,413 \\
\hline $\begin{array}{l}\text { GORDox } 5 \min (\%) \\
\text { GORDox } 30 \mathrm{~min}(\%)\end{array}$ & $\begin{array}{l}16,67 \pm 10,74 \\
12,85 \pm 11,43\end{array}$ & $-0,867$ & 0,408 \\
\hline
\end{tabular}

CHOox (\%) Total de carboidratos oxidados nos 30 min de exercícios; GORDox (\%): Total de gordura oxidada nos 30 min de exercícios; CHOox 5 min (\%): Carboidrato oxidado nos primeiros 5 min; $\mathrm{CHO}$ ox $5 \mathrm{~min}$ (\%): Carboidrato oxidado nos últimos $5 \mathrm{~min}$; GORDox 5 min (\%): Gordura oxidada nos primeiros $5 \mathrm{~min}$; GORDox $30 \mathrm{~min}$ (\%): Gordura oxidada nos últimos 5 min; ${ }^{*} p<0,05$.

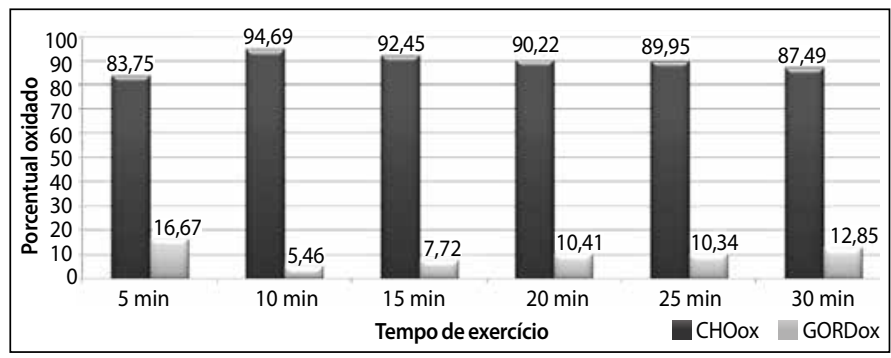

Figura 1. Percentual de oxidação de substratos energéticos a cada 5 min de exercício.

Tabela 4. Correlações entre as variáveis.

\begin{tabular}{c|c|c|c|c|c|c|c|c}
\hline & HbA1c & VO $^{2}$ & IMCz & $\begin{array}{c}\text { GLIC. } \\
\text { PRE் }\end{array}$ & $\begin{array}{c}\text { GLIC. } \\
\text { PÓS }\end{array}$ & $\begin{array}{c}\text { GLIC. } \\
\mathbf{3 0 m i n} \\
\text { PÓs }\end{array}$ & $\begin{array}{c}\text { CHO } \\
\text { Ox(\%) }\end{array}$ & $\begin{array}{c}\text { GORD } \\
\text { ox(\%) }\end{array}$ \\
\hline HbA1C & 1,00 & $-0,480$ & $0,821^{* *}$ & $0,815^{* *}$ & $0,702^{*}$ & 0,351 & $-0,407$ & 0,411 \\
\hline VO $_{\text {máx }}^{2}$ & & 1,00 & $-0,610$ & $-0,391$ & $-0,118$ & 0,271 & $-0,451$ & 0,447 \\
\hline IMCz & & & 1,00 & 0,544 & 0,472 & 0,188 & $-0,068$ & 0,072 \\
\hline GLIC.PRÈ & & & & 1,00 & $0,851^{* *}$ & 0,589 & $-0,210$ & 0,214 \\
\hline GLICPOS & & & & 1,00 & $0,874^{* *}$ & $-0,323$ & 0,325 \\
\hline $\begin{array}{c}\text { GLIC.30min. } \\
\text { PÓS }\end{array}$ & & & & & 1,00 & $-0,355$ & 0,356 \\
\hline CHOOX (\%) & & & & & & & 1,00 & $-1,000^{* *}$ \\
\hline
\end{tabular}

HbA1c: Hemoglobina glicosilada; $\mathrm{VO}_{2 \text { maxx }}$ Capacidade cardiorrespiratória; IMCz: Índice de massa corporal score z; GLIC. PRÉ: Glicose pré-exercício; GLIC.POS: Reduçăo da glicose sanguínea ao final do exerć́cio; GLIC.30min. PÓs: Glicose 30 min após término do exercício; $\mathrm{CHOox}(\%)$ : Carboidrato oxidado durante o exercício; GORDox (\%): Gordura oxidada durante o exercício; ${ }^{* *} P<0,01$. ${ }^{*} p<0,05$.

Observando a glicemia avaliada 30 minutos após o término dos exercícios, podemos notar um pequeno aumento dos níveis glicêmicos

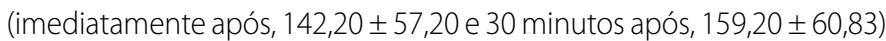
demonstrando um efeito hiperglicemiante no período de recuperação logo após o exercício.

O estudo de Guelfi et al.7 comparou protocolos de exercícios contínuos com exercícios intermitentes observaram uma menor diminuição da glicemia pós exercícios nos protocolos com exercícios intermitentes com uma redução média de 18,18\% da glicemia, este resultado foi associado a maiores níveis de lactato e catecolaminas induzidas por exercícios intermitentes. Essa resposta glicêmica que favorece a um menor risco de hipoglicemias induzidas pelos exercícios tem sido associado a uma maior produção endógena de glicose, em outro estudo de Guelfi et al. ${ }^{8}$ aonde avaliaram a produção endógena de glicose com dois protocolos de exercícios intermitentes e contínuos, os autores encontraram resultados interessantes com uma maior produção endógena de glicose no grupo que realizou exercícios intermitentes.

Outro trabalho de Iscoe e Riddell ${ }^{13}$ avaliou a glicemia de 11 atletas treinados com exercícios com ou sem sprints máximos e suas respostas na glicemia até 48 h após a realização, os resultados demonstraram menor risco de hipoglicemias noturnas no grupo com exercícios intermitentes e que esse tipo de exercícios pode ser uma estratégia interessante para diminuir os riscos de hipoglicemias induzidas pelo exercício.

O presente estudo descritivo demonstra uma diminuição da glicemia (21\%) com tendência de aumento no período inicial de recuperação, o que pode ser uma estratégia interessante para evitar os riscos de hipoglicemias durante e logo após a prática.

Essa pequena diminuição ou até mesmo uma hiperglicemia induzida após os exercícios intermitentes de alta intensidade está associada ao aumento da secreção dos hormônios contrarreguladores como glucagon, catecolaminas e cortisol, ocasionando aumento da produção de glicose pelo fígado e liberação de ácidos graxos ${ }^{14}$.

Durante a prática de exercícios físicos a um aumento da atividade da AMPK em resposta a contração muscular, e consequentemente aumento da captação de glicose para dentro do músculo para gerar ATP, aumentando a translocação de GLUT-4 para a membrana celular ${ }^{15}$. Os exercícios aeróbicos exigem uma necessidade predominante da utilização de 
oxigênio, para obter a oxidação de substratos energéticos principalmente carboidratos e gorduras e a proporção destes substratos que serão oxidados depende diretamente da intensidade do exercício ${ }^{16}$. Ao analisar os substratos utilizados durante os exercícios no presente estudo, observa-se que pequenos sprints máximos de apenas 10 segundos realizados a cada 5 minutos, estimula o metabolismo a uma tendência predominante da oxidação de $\mathrm{CHO}$ e não de gorduras como principal fonte energética, desde os primeiros 5 minutos até o término do exercício, segundo Colberg ${ }^{17}$ a utilização de carboidratos como fontes energéticas aumenta rapidamente quando você começa a exercitar-se ou quando há um incremento na intensidade, ainda os exercícios de intensidades altas ou quase máximas utilizam praticamente $100 \%$ de carboidratos e $0 \%$ de gordura.

Ocorreu uma correlação positiva forte entre a HbA1c e o IMC escore $z(r=0,821)$ e entre HbA1c e glicose observada pós-exercício $(r=0,702)$. Então com base nestes resultados podemos observar que quanto maior o IMC escore z dos pacientes, pior é seu controle glicêmico observado por HbA1c, demonstrando a importância de os diabéticos manter o peso corporal dentro dos parâmetros desejados, para isso é importante a manutenção de uma prática regular de atividades físicas e uma alimentação saudável para se obter um bom controle glicêmico e redução do risco de doenças cardiovasculares ${ }^{18}$.

É importante a manutenção de um bom controle glicêmico, pois níveis constantes de HbA1c acima de 7\% já apresentam maiores riscos de ter complicações a longo prazo como complicações micro e macrovasculares ${ }^{19}$. Segundo Nathan et al. ${ }^{20}$ valores de HbA1 c acima de 10,4\% estão associadas a um aumento de sete vezes o risco de morte devido a complicações cardiovasculares. Um estudo recente demonstrou que valores de $\mathrm{HbA} 1 \mathrm{c}$ menores que $7,6 \%$ esta associada a uma prevenção do desenvolvimento de complicações microvasculares por no mínimo 20 anos $^{21}$.

Há uma correlação direta forte entre os níveis de glicose pré-exercício e pós-exercício ( $r=0,851$, $p=0,002)$. Ou seja, quanto maior for o nível glicêmico inicial maior será a glicemia ao término do mesmo. É importante ressaltar que os limites para uma prática segura de exercícios físicos inclusive para atletas com diabetes é uma glicemia inicial entre 140 e 250 mg/dl22.

$O$ teste de regressão linear mostrou que o IMC escore z explica $67 \%$ dos valores de $\mathrm{HbA} 1 \mathrm{c}\left(\mathrm{r}^{2}=0,675\right)$. Isso só reafirma a importância de adolescentes diabéticos se engajarem cada vez mais na prática de exercícios físicos. É importante que práticas educativas sejam implantadas por equipes multidisciplinares para conscientizar a população da importância de uma alimentação saudável e da prática regular de atividade física, a fim de auxiliar no controle de peso corporal, controle metabólico e diminuição dos riscos de complicações agudas de diabéticos ${ }^{18}$.

O presente estudo apresenta algumas limitações como a ausência de um grupo controle, o número amostral $(n=10)$ que pode comprometer a generalização dos resultados para a população estudada. Não foram controladas possíveis variáveis de confusão como estágio de maturação sexual e possíveis alterações hormonais associadas, então as associações apresentadas devem ser interpretadas com cautela.

Futuros estudos devem ser realizados utilizando grupos controles e análises das respostas hormonais induzidas pelo exercício, com o intuito de esclarecer melhor os efeitos dos exercícios intermitentes na resposta glicêmica e alterações metabólicas de crianças e adolescentes, bem como seus benefícios e possíveis riscos. Mais estudos são importantes para fornecer dados mais concretos para que crianças e adolescentes possam aproveitar com segurança da prática dos exercícios intermitentes, tendo em vista que estes possuem características semelhantes aos diversos esportes e brincadeiras espontâneas comumente praticadas.

\section{CONCLUSÃO}

De acordo com os resultados observados, 30 minutos de exercícios aeróbicos intermitentes intercalados com tiros curtos de 10 segundos promovem uma redução média de $21 \%$ da glicemia com tendência de aumento na fase de recuperação. O substrato energético predominante na atividade são os carboidratos $(\mathrm{CHO})$.

\section{AGRADECIMENTOS}

Os autores agradecem ao CNPq e CAPES pelo financiamento da pesquisa e bolsas de estudo.

Todos os autores declararam não haver qualquer potencial conflito de interesses referente a este artigo.

CONTRIBUIÇÕES DOS AUTORES: Cada autor contribuiu individual e significativamente para o desenvolvimento do manuscrito. VAL (0000-0002-9413-4645)* e LPGM $(0000-0002-7762-2727)^{*}$ foram os principais contribuintes na redação do manuscrito. WCS (000-0002-1585-0353)*, JPD (0000-0002-4132-8809)* e SNF (0000-0002-3987-3998)* participaram da coleta de dados clínicos e testes realizados com os pacientes. NL (0000-0002-4752-6697)* orientou e coordenou todo o projeto e pesquisa além da revisão do manuscrito. Todos os autores participaram ativamente na discussão dos resultados, revisão e aprovação da versão final do trabalho. *ORCID (Open Researcher and Contributor ID).

\section{REFERÊNCIAS}

1. Mercuri N, Arrechea V. Atividade física e diabetes mellitus. Diabetes Clínica. 2001;5(5):347-9.

2. Canadian Diabetes Association Clinical Practice Guidelines Expert Committee, Cheng AY. Canadian Diabetes Association 2013 clinical practice guidelines for the prevention and management of diabetes in Canada. Introduction. Can J Diabetes. 2013 Apr;37 Suppl 1:S1-3.

3. Francescato MP, Geat M, Fusi S, Stupar G, Noacco C, Cattin L. Carbohydrate requirement and insulin concentration during moderate exercise in type 1 diabetic patients. J Metabol. 2004;53(9):1126-30.

4. Campaigne BN; Wallberg-Henriksson $\mathrm{H}$; Gunnarsson R. Glucose and insulin responses in relation to insulin dose and caloric intake $12 \mathrm{~h}$ after acute physical exercise in men with IDDM. Diabetes Care. 1987;10(6):716-21.

5. Bailey RC1, Olson J, Pepper SL, Porszasz J, BarstowTJ, Cooper DM. The level and tempo of children's physical activities: an observational study.. Med Sci Sports Exerc. 1995;27(7):1033-41.

6. Raile K, Kapellen T, Schweiger A, Hunkert F, Nietzschmann U, Dost A, et al. Physical activity and competitive sports in children and adolescents with type 1 diabetes.. Diabetes Care. 1999;22(11):1904-5.

7. Guelfi KJ, Jones TW, Fournier PA. The decline in blood glucose levels is less with intermittent high-intensity compared with moderate exercise in individuals with type 1. Diabetes Care. 2005;28(6):1289-94.

8. Guelfi KJ, Ratnam N, Smythe GA, Jones TW, Effect of intermittent high-intensity compared with continuous moderate exercise on glucose production and utilization in individuals with type 1 diabetes. Am J Physiol Endocrinol Metab. 2007;292(3):865-70

9. Lohman TG. Advances in body composition assessment: current issues in exercise science. Monograph Champaign: Human Kinetics Publishers; 1992.

10. Rowland TW. Exercise and children's health. Champaign: Human Kinetics Books; 1990.

11. Tanaka H, Monahan KD, Seals DR. Age-predicted maximal heart rate revisited. J Am Coll Cardiol. 2001;37(1):153-6.
12. Institute of Medicine (IOM). Dietary reference intakes: for Energy, Carbohydrate, Fiber, Fat, Fatty Acids, Cholesterol, Protein, and Amino Acids (Macronutrients). Washington, DC: National Academy Press; 2005.

13. Iscoe KE, Riddell MC. Continuous moderate-intensity exercise with or without intermittent high-intensity work: effects on acute and late glycaemia in athletes with Type 1 diabetes mellitus. Diabet Med. 2011;28(7):824-32.

14. Kitabchi AE, Umpierrez GE, Murphy MB, Barrett EJ, Kreisberg RA, Malone Jl, et al. Hyperglycemic crises in diabetes. Diabetes Care. 2004;27:(Suppl 1): S94-102.

15. Ropelle ER, Pauli JR, Carvalheira JBC. Efeitos moleculares do exercício físico sobre as vias de sinalização insulínica. Motriz. 2005;11(1):49-55.

16. Sasaki JE, Santos MG. O papel do exercício aeróbico sobre a função endotelial e sobre os fatores de risco cardiovasculares. Arq Bras Cardiol. 2006;87(5):e227-33.

17. Colberg Sheri. Atividade física e diabetes. Barueri: Manole. 2003; p. 25.

18. Rique $A B R$, Soares EA, Meirelles CM. Nutrição e exercício na prevenção e controle das doenças cardiovasculares. Rev Bras Med Esporte. 2002;8(6):244-54

19. Lehto S, Ronnemaa T, Pyorala K, Laakso M. Poor glycemic control predicts coronary heart disease events in patients with type 1 diabetes without nephropathy. Arterioscler Thromb Vasc Biol. 1999;19:1014-9.

20. Nathan DM, Lachin J, Cleary P, Orchard T, Brillon DJ, Backlund JY, et al. Intensive diabetes therapy and carotid intima-thickness in type 1 diabetes mellitus. N Engl J Med; 2003;348(23):2294-303.

21. Nordwall M, Abrahamsson M, Dhir M, Fredrikson M, Ludvigsson J, Arnqvist HJ. Impact of HbA1c, Followed From Onset of Type 1 Diabetes, on the Development of Severe Retinopathy and Nephropathy: The VISS Study (Vascular Diabetic Complications in Southeast Sweden). Diabetes Care; 2015;38(8):e124.

22. Sociedade Brasileira de Diabetes. Protocolo hiper/hipoglicemias x corrida para atletas com diabetes. [Internet] 2015 [acesso em 2016 abr 13]. Disponivel em: http://www.diabetes.org.br/protocolos/protativfis.php 